\title{
Elementary Excitations in One-Dimensional Electromechanical Systems; Transport with Back-Reaction
}

\author{
Kang-Hun $\mathrm{Ahn}^{1}$ and Hangmo $\mathrm{Yi}^{2}$ \\ 1 Department of Physics, Chungnam National University, Daejon 305-764, Korea \\ ${ }^{2}$ School of Physics, Korea Institute for Advanced Study, Seoul 130-722, Korea
}

(Dated: November 21, 2018)

\begin{abstract}
Using an exactly solvable model, we study low-energy properties of a one-dimensional spinless electron fluid contained in a quantum-mechanically moving wire located in a static magnetic field. The phonon and electric current are coupled via Lorentz force and the eigenmodes are described by two independent boson fluids. At low energies, the two boson modes are charged while one of them has excitation gap due to back-reaction of the Lorentz force. The theory is illustrated by evaluating optical absorption spectra. Our results are exact and show a non-perturbative regime of electron transport.

PACS numbers: 73.23.-b, 71.10.Pm, 72.15.-v
\end{abstract}

Electronic and mechanical properties of nanoelectromechanical systems (NEMS) are recently of fundamental and technical interest [1]. Perpetual trend of miniaturization of electronic devices has already led NEMS smaller down to molecular scale, e.g., nanotube electromechanical systems [2] which are likely the bases of new novel devices in NEMS application [3]. These systems are close to the regime of quantum-mechanical operation which is the ultimate limit of nanoelectromechanical devices. For the design of quantum mechanically operating NEMS devices, it is crucial to understand first the quantum effects of mechanical motion coupled to electric current. Here we ask ourselves; Are the electric currents influenced by the quantum nature of mechanical motion? If so, what is the signature of the quantum fluctuation?

To answer these, we study one-dimensional spinless electron fluids coupled to their quantum-mechanical motion in a static magnetic field. This model is exactly solvable as will be shown here. In this way, we could describe both the electrical and mechanical degrees of freedom in a purely quantum mechanical manner on an equal footing. The harmonic-fluid approach used for electron fluids in a static wire, which are referred to as (Tomonaga-)Luttinger liquid theory [4, 5, 6], could be successfully applied to describe the electric currents coupled to mechanical motions through Lorentz force. This magneto-electro-mechanical coupling is essential in socalled electromotive-force technique which is popularly used in NEMS-based force sensors [7, [8].

In this paper, we show that electric currents in nanoelectromechanical wires are strongly influenced by the quantum fluctuations of mechanical motion through the magneto-electro-mechanical coupling. This coupling causes interesting and peculiar phenomena because of the feedback reaction between electric and mechanical movement. As we shall see, an important frequency scale related to a gap structure arises due to this feedback reaction. The feature we found could be observed generally in other vibrating one-dimensional electronic systems such as atomic wires 9 and quasi-one-dimensional metals 10 .

We model the electrons with an interacting spinless Fermion fluid with a periodic boundary condition $\Psi(x+$ $L)=\Psi(x)$. These Fermions are contained in a wire of mass density $\rho_{m}$ and tension $\mathcal{T}$, and a magnetic field $\mathbf{B}$ is applied perpendicular to $\mathrm{x}$-axis. The displacement $u(x)$ is in the direction of $\hat{\mathbf{x}} \times \mathbf{B}$, and assumed small $\left(\partial_{x} u \ll 1\right)$ and periodic $(u(x+L)=u(x))$. The electrons and the wire are assumed strongly tightened so any force exerted on the electrons in $\mathrm{u}$-direction is delivered to the wire immediately. The Hamiltonian reads $(\hbar=1, e<0)$

$$
\begin{aligned}
H & =\frac{1}{2 m} \int d x \Psi^{\dagger}(x)\left(\partial_{x}-i e A_{x}\right)^{2} \Psi(x) \\
& +\frac{1}{2} \int d x d x^{\prime} V\left(x-x^{\prime}\right) \rho(x) \rho\left(x^{\prime}\right)+H_{u} \\
H_{u} & =\frac{1}{2} \int d x\left[\frac{1}{\rho_{m}} \pi_{u}(x)^{2}+\mathcal{T}\left(\partial_{x} u(x)\right)^{2}\right] .
\end{aligned}
$$

Here $\pi_{u}$ is the conjugate momentum of $u(x)$; $\left[u(x), \pi_{u}\left(x^{\prime}\right)\right]=i \delta\left(x-x^{\prime}\right) . \quad A_{x}=B u(x)+\Phi_{B} / L$ is the gauge potential for the applied magnetic field where $\Phi_{B}$ is a constant magnetic flux enclosed by the circle of radius $L / 2 \pi$. For the sake of convenience, let us redefine $u(x)+\Phi_{B} / B L \rightarrow u(x)$ so that $A_{x}=B u(x)$.

Following the approach of Haldane $[\underline{6}]$, we use a Boson field $\theta$ defined from the Fermion density $\rho(x)=$ $\left(k_{F}+\partial_{x} \theta\right) / \pi$ and its conjugate field $\phi\left(\left[\phi(x), \theta\left(x^{\prime}\right)\right]=\right.$ $\left.i \frac{1}{2} \pi \operatorname{sgn}\left(x-x^{\prime}\right)\right)$. The boson fields describe the long wavelength fluctuations of the Fermion field $\Psi^{\dagger}(x)$. It proves convenient to define new Fermion field $\psi^{\dagger}=\exp (i \Lambda) \Psi^{\dagger}$, where $\Lambda(x)=e B \int^{x} u\left(x^{\prime}\right) d x^{\prime}$. In terms of $\theta, \phi$ and $\Lambda$, the new Fermion field $\psi^{\dagger}$ is written as

$$
\psi^{\dagger}(x) \sim \sum_{\mathrm{n} \text { odd }} \exp \left[\operatorname{in}\left[\theta(x)+k_{F} x\right]\right] \exp [i(\phi(x)+\Lambda(x))](2)
$$

The boundary conditions for the Boson fields are $\theta(x+$ $L)=\theta(x)+\pi \mathrm{N}, \phi(x+L)=\phi(x)+\pi \mathrm{J}$, and $\Lambda(x+L)=$ $\Lambda(x)+e B u_{0} L$, where $u_{0}=\frac{1}{L} \int_{0}^{L} d x u(x)$, and the sum of 
the topological numbers $\mathrm{N}$ and $\mathrm{J}$ is an even integer. Note that the new Fermion field now has a twisted boundary condition $\psi(x+L)=\exp \left(-i e B u_{0} L\right) \psi(x)$.

The effective Hamiltonian describing long-distance physics is given by taking terms of $n= \pm 1$ in Eq.(2) with a proper normalization constant;

$$
H=\frac{v}{2 \pi} \int d x\left(g\left(\partial_{x} \phi+e B u\right)^{2}+g^{-1}\left(\partial_{x} \theta\right)^{2}\right)+H_{u} .
$$

Here the dimensionless constant $g$ is governed by electron-electron interaction strength and $v=v_{F} / g$ is the electronic wave velocity, where $v_{F}$ is the Fermi velocity for the non-interacting case. For short-ranged electron interactions $V\left(x-x^{\prime}\right)=V \delta\left(x-x^{\prime}\right), g$ is given by $1 / \sqrt{1+V / \pi v_{F}}$. In terms of the boson fields, the electrical current density is given by

$$
J(x)=-e v_{F} \partial_{x} \phi(x)-v_{F} e^{2} B u(x) .
$$

Note that the term of $u^{2}$ in Eq.(3) and $H_{u}$ describe phonons of finite mass at small momenta;

$$
\frac{g v}{2 \pi} e^{2} B^{2} \int d x u^{2}(x)+H_{u}=\sum_{q} \tilde{\omega}_{s}(q) a_{q}^{\dagger} a_{q},
$$

Here

$$
\tilde{\omega}_{s}(q)=\sqrt{v_{s}^{2} q^{2}+\omega_{B}^{2}},
$$

where $v_{s}=\sqrt{\mathcal{T} / \rho_{m}}$ is the bare sound velocity and $\omega_{B}=\sqrt{\frac{v_{F} e^{2}}{\pi \rho_{m}}} B$. The first term in Eq. (55) essentially gives mass to phonon and originates from back-reaction process in the system. Note that when the wire moves, an induced electric field $\mathcal{E}_{\text {emf }}=-B \partial_{t} u$ changes the momentum of electrons by $\int d t e \mathcal{E}_{\text {emf }}=-e B u$, which causes the change in electric current $-e^{2} B u \rho_{0} / m$ where $\rho_{0}=k_{F} / \pi$ is the mean Fermion density. The Lorentz force $-v_{F} e^{2} B^{2} u / \pi$ exerted on the induced electric current again acts on the movement of the wire, which we call here the back-reaction process.

Now let us use the following Fourier-transformed field operators;

$$
\begin{aligned}
& \theta(x)=\theta_{0}+\frac{\pi \mathrm{N} x}{L}-i \sum_{q \neq 0} \sqrt{\frac{g \pi}{2|q| L}}\left(b_{q}^{\dagger}+b_{-q}\right) \operatorname{sgn}(q) e^{i q x} \\
& \phi(x)=\phi_{0}+\frac{\pi \mathrm{J} x}{L}-i \sum_{q \neq 0} \sqrt{\frac{\pi}{2 g|q| L}}\left(b_{q}^{\dagger}-b_{-q}\right) e^{i q x} \\
& u(x)=u_{0}+\sum_{q \neq 0} \sqrt{\frac{1}{2 \rho_{m} \tilde{\omega}_{s}(q) L}}\left(a_{-q}^{\dagger}+a_{q}\right) e^{i q x} \\
& \pi_{u}(x)=\frac{\pi_{u 0}}{L}+i \sum_{q \neq 0} \sqrt{\frac{\rho_{m} \tilde{\omega}_{s}(q)}{2 L}}\left(a_{-q}^{\dagger}-a_{q}\right) e^{i q x}
\end{aligned}
$$

Here $\left(\theta_{0}, \mathrm{~J}\right)$ and $\left(\phi_{0}, \mathrm{~N}\right)$ are pairs of conjugate action-angle variables $[6]$ and $\left[u_{0}, \pi_{u 0}\right]=i$.

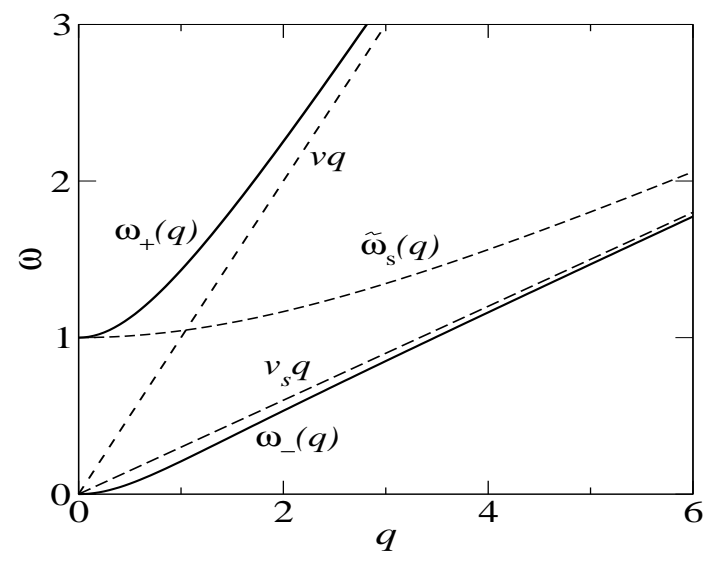

FIG. 1: The dispersion relation of the eigenmodes $\omega_{ \pm}(q)$ (solid lines). $\omega$ is in unit of $\omega_{B}$ and $q$ is in unit of $\omega_{B} / v$. The dotted lines denote the boson mode for electron $\omega=v q$, phonon $\omega=v_{s} q$, and the massive phonon $\tilde{\omega}_{s}(q)$. We chose $v_{s}=0.3 v$ in this figure.

Using these operators, the Hamiltonian in Eq.(3) is written as

$$
\begin{aligned}
H & =\sum_{q} v|q| b_{q}^{\dagger} b_{q}+\sum_{q} \tilde{\omega}_{s}(q) a_{q}^{\dagger} a_{q}+H_{Z M} \\
& +\frac{\omega_{B}}{2} \sum_{q} \sqrt{\frac{v|q|}{\tilde{\omega}_{s}(q)}}\left(b_{-q}^{\dagger}-b_{q}\right)\left(a_{q}^{\dagger}+a_{-q}\right) \operatorname{sgn}(q)
\end{aligned}
$$

Here $H_{Z M}$ describes the zero-mode; $H_{Z M}=\frac{\pi v_{F}}{2 L}(\mathrm{~J}+$ $\left.\frac{e B u_{0} L}{\pi}\right)^{2}+\frac{\pi_{u 0}^{2}}{2 \rho_{m} L}+\frac{v \pi}{2 g L} \mathrm{~N}^{2}$, according to which the centerof-mass of the wire executes a simple harmonic oscillation with resonance frequency $\omega_{B}$.

The Hamiltonian in Eq. (8) )is now diagonalized using new Boson operators $\gamma_{q, \pm}$, which are linear combinations of the four boson operators $b_{q}, b_{-q}^{\dagger}, a_{q}$, and $a_{-q}^{\dagger}$;

$$
H=\sum_{q, \pm} \omega_{ \pm}(q) \gamma_{q, \pm}^{\dagger} \gamma_{q, \pm}+H_{Z M}
$$

where the dispersion relations for the two eigenmodes are

$$
\begin{aligned}
& \omega_{ \pm}(q)= \\
& \sqrt{\frac{\left(v^{2}+v_{s}^{2}\right) q^{2}+\omega_{B}^{2} \pm \sqrt{\left.\left(v^{2}-v_{s}^{2}\right) q^{2}-\omega_{B}^{2}\right)^{2}+4 \omega_{B}^{2} v^{2} q^{2}}}{2}} .
\end{aligned}
$$

Now the ground state is the vacuum of new bosons, $\gamma_{q \pm} \mid 0>=0$.

For detailed discussion, let us assume $v>v_{s}$ which is usually the case. In Fig. 1, we plot dispersion relations for the two modes. As $q$ increases or $B$ decreases, the system goes to a weak coupling regime where the ' + ' mode becomes electronic $\left(\gamma_{q+} \sim b_{q}, \omega_{+}(q) \sim v q\right)$ and the ${ }^{\prime}{ }^{\prime}$ mode becomes phononic $\left(\gamma_{q-} \sim a_{q}, \omega_{-}(q) \sim\right.$ $\left.\tilde{\omega}_{s}(q)\right)$. In the opposite limit where $q$ is small and $B \neq$ 0 , the system is in a strong coupling regime where the 
two uncoupled modes undergo level crossing as shown in Fig. 1 and eventually completely exchange their identity in $q \rightarrow 0$ limit, i.e., $\gamma_{q,+} \rightarrow a_{q}$ and $\gamma_{q,-} \rightarrow b_{q}$. It is instructive to note that electric current can flow through the ' + ' modes even in $q \rightarrow 0$ limit, where the ' + ' modes become phononic. This is because of the 'diamagnetic' contribution of electric current $-v_{F} e^{2} B u(x)$ in Eq.(4).

In the strong coupling regime, the dispersion relation of the ' + ' mode is in the same form with plasmon dispersion relation, $\omega_{+}(q) \sim \sqrt{v^{2} q^{2}+\omega_{B}^{2}}\left(q \ll \omega_{B} / v\right)$. In contrast to the plasmon [11], however, the frequency gap $\omega_{B}$ originates from the back-reaction which gives mass to phonon at long-wavelength limit, $\tilde{\omega}_{s}(q) \rightarrow \omega_{B}$. The '-' mode is gapless $\omega_{-}(q) \sim v_{s} v q^{2} / \omega_{B}\left(q \ll \omega_{B} / v_{s}\right)$. This mode is also charged at small $q$, where electronic and phononic excitation are entangled with each other. These excitations at long wavelengths are non-perturbative and can not be captured by perturbative expansions in $B$.

Now let us consider experiments where the mechanical quantum fluctuations can be observed. In Fig. 2, we depict a system we consider. An external electrical field $V_{e}(t) / d$ is applied along the wire in $0<x<d$ by nearby external metallic gates with oscillating voltage difference $V_{e}(t)=V_{e}(\omega) e^{i \omega t}$. If the current $I(\omega)$ is measured far away from the regime $0<x<d$, the current will not be sensitive to the position of the contacts so can be given by its averaged value $I(\omega) \approx \frac{1}{d} \int_{0}^{d} d x J(x, \omega)$. From the linear response theory, one gets

$$
\alpha(\omega) \equiv \frac{I(\omega)}{V_{e}(\omega)}=\frac{1}{i \omega d^{2}} \int_{0}^{d} d x \int_{0}^{d} d x^{\prime} \Pi\left(x, x^{\prime}, \omega\right),
$$

where $\Pi\left(x, x^{\prime} \omega\right)=i \int_{0}^{\infty} d s<\left[J(x, 0), J\left(x^{\prime},-s\right)\right]>e^{i \omega s}$ is the current-current correlation function.

This form is equivalent to the conductance formula $G(\omega)$ used by Kane and Fisher 12 but caution is needed here. In our case, additional electromotive force $V_{\text {emf }}(\omega)$ is induced along the wire when $B \neq 0$. In usual twoterminal conductance measurements, since the measured voltage $V(\omega)$ is given by $V_{\mathrm{emf}}(\omega)+V_{e}(\omega)$, the twoterminal conductance is related to our $\alpha(\omega)$ as $I(\omega)=$ $G(\omega)\left(V_{e}(\omega)+V_{\text {emf }}(\omega)\right)=\alpha(\omega) V_{e}(\omega)$.

The real part of $\alpha(\omega)$ should be understood as an optical absorption spectrum. After averaging over a time period $2 \pi / \omega$, the power $P(\omega)$ absorbed from the external electric field to the wire is given by $P(\omega)=$ $\frac{1}{2} \operatorname{Re} \int_{0}^{d} d x\left(V_{e}^{*}(\omega) / d\right) J(x, \omega)=\frac{1}{2} \operatorname{Re} \alpha(\omega)\left|V_{e}(\omega)\right|^{2}$.

To evaluate conveniently the current correlation function, we switch to a Lagrangian formalism following Kane and Fisher 12. The Hamiltonian in Eq.(33) is equivalent to the following Euclidean action,

$$
\begin{aligned}
S & =\frac{v}{2 g} \int d \tau d x\left(\left(\partial_{x} \theta\right)^{2}+\frac{1}{v^{2}}\left(\partial_{\tau} \theta\right)^{2}\right) \\
& +\frac{\mathcal{T}}{2} \int d \tau d x\left(\left(\partial_{x} u\right)^{2}+\frac{1}{v_{s}^{2}}\left(\partial_{\tau} u\right)^{2}\right)
\end{aligned}
$$

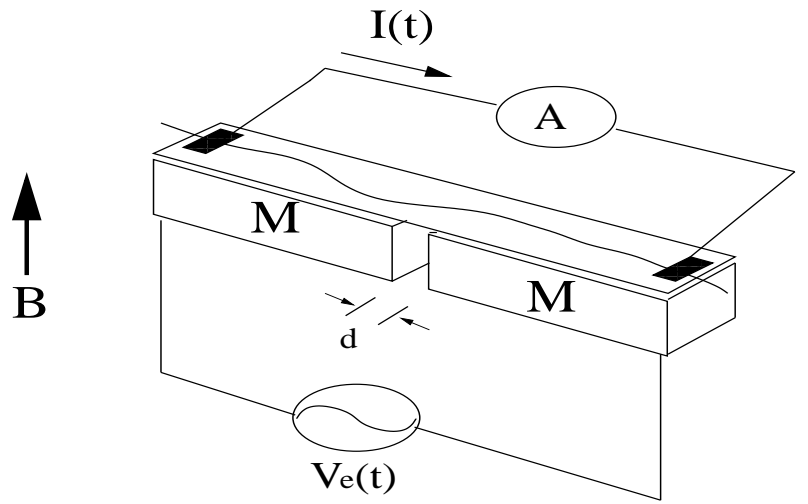

FIG. 2: Schematic figure for the experimental set-up in consideration. The wire is on an insulating layer above two metallic gates denoted by $\mathrm{M}$.

$$
\text { - } B \int d \tau d x J(x, \tau) u(x, \tau)
$$

where $J(x, \tau)=i e \partial_{\tau} \theta(x, \tau) / \pi$. Evaluation of the current correlation function $\Pi\left(x, x^{\prime}, i \omega_{n}\right)$ at Matsubara frequencies $\omega_{n}=2 \pi n / \beta$ can be easily done using the above Euclidean action;

$$
\begin{aligned}
& \Pi\left(x, x^{\prime}, i \omega_{n}\right)=\int_{0}^{\beta} d \tau e^{-i \omega_{n} \tau}<\mathrm{T}_{\tau} J(x, \tau) J\left(x^{\prime}, 0\right)> \\
= & -\frac{e^{2} v_{F}}{2 \pi^{2}} \int_{-\infty}^{\infty} d q e^{i q\left(x-x^{\prime}\right)} \frac{\omega_{n}^{2}}{q^{2} v^{2}+\omega_{n}^{2}+\omega_{B}^{2} \frac{\omega_{n}^{2}}{q^{2} v_{s}^{2}+\omega_{n}^{2}}} .(13)
\end{aligned}
$$

Now, by performing analytic continuation $\Pi\left(x, x^{\prime}, \omega\right)=$ $\Pi\left(x, x^{\prime}, i \omega_{n} \rightarrow \omega+0^{+}\right)$, we get $\operatorname{Re} \alpha(\omega)$ from Eq.(11);

$$
\begin{aligned}
& \operatorname{Re} \alpha(\omega) \\
= & \frac{e^{2}}{4 \pi} v_{F}|\omega| \int_{-\infty}^{\infty} d q \frac{\sin ^{2}(q d / 2)}{(q d / 2)^{2}} \frac{\omega^{2}-\left(q v_{s}\right)^{2}}{\omega^{2}-\left(\omega_{+}^{2}(q)+\omega_{-}^{2}(q)\right) / 2} \\
\times & \left\{\delta\left(\omega^{2}-\omega_{+}^{2}(q)\right)+\delta\left(\omega^{2}-\omega_{-}^{2}(q)\right)\right\} .
\end{aligned}
$$

The result of the above integration is written in terms of dimensionless parameters $\tilde{\omega}=|\omega| d / v_{s}, \tilde{B}=L \omega_{B} / v_{s}$, and $\eta=v_{s} / v ; \operatorname{Re} \alpha(\omega)=g \frac{e^{2}}{h} \sum_{ \pm} A_{ \pm}(\tilde{\omega}, \tilde{B}, \eta)$. (Here we revived the Planck constant.) The dimensionless function $A_{ \pm}$are

$$
A_{ \pm}= \pm \frac{\sin ^{2}\left(\kappa_{ \pm} / 2\right)}{\left(\kappa_{ \pm} / 2\right)^{2}} \frac{\eta\left(\tilde{\omega}^{2}-\kappa_{ \pm}^{2}\right) / \kappa_{ \pm}}{\sqrt{\left(1-\eta^{2}\right)^{2} \tilde{\omega}^{2}+4 \tilde{B}^{2}}}
$$

where $\kappa_{ \pm}=\sqrt{\left(\left(1+\eta^{2}\right) \tilde{\omega}^{2} \mp \tilde{\omega} \sqrt{\left(1-\eta^{2}\right)^{2} \tilde{\omega}^{2}+4 \tilde{B}^{2}}\right) / 2}$.

In Fig. 3, we plot the field absorption power $\operatorname{Re} \alpha(\omega)$ using the exact formula we obtained. Since usually $v_{s} \ll$ $v$ (for metallic carbon nanotubes, $v_{s} \sim 15 \mathrm{~km} / \mathrm{s} \ll v$ and $v \sim 10^{5} \mathrm{~km} / \mathrm{s}$ ), let us assume $\eta \ll 1$. In this case, the induced electric current is strongly suppressed when 


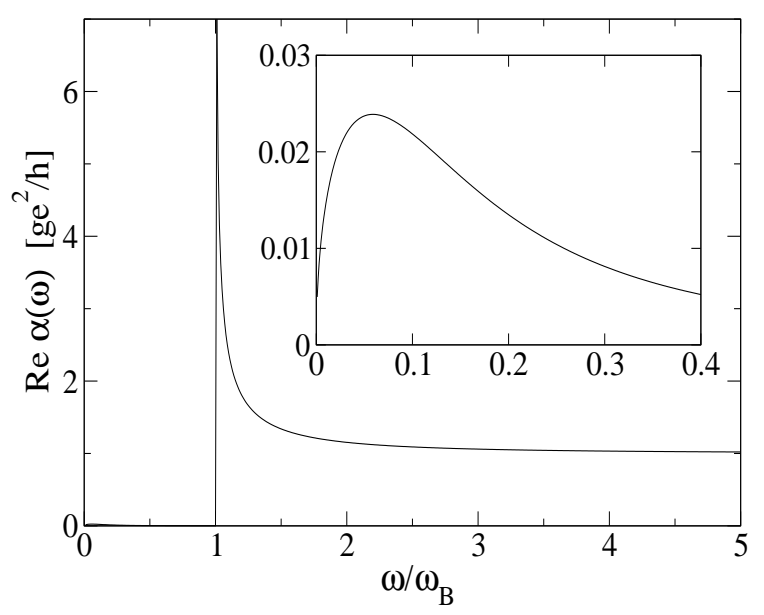

FIG. 3: The optical absorption spectrum for $v_{s} / v=0.1$ and $\omega_{B} d / v=10^{-4}$. The inset shows the details at low frequencies. In the low frequency regime (inset), the maximum arises near $\omega \approx\left(v_{s} / v\right) \omega_{B}$.

$|\omega|<\omega_{B}$. There are three interesting frequency ranges. (i) $\omega \ll\left(v_{s} / v\right) \omega_{B}$. Here only '-' modes are excited which are mainly electronic density fluctuations. In this regime, using $A_{-} \approx \frac{\eta}{2} \sqrt{\frac{\tilde{\omega}}{\tilde{B}}} \ll 1$ and $A_{+}=0$, we get

$$
\operatorname{Re} \alpha(\omega) \approx g \frac{e^{2}}{h} \frac{\sqrt{v_{s} / v}}{2} \sqrt{\frac{\omega}{\omega_{B}}}
$$

It is interesting to note the anomalous frequency and magnetic-field dependence $\operatorname{Re} \alpha(\omega) \propto \sqrt{\omega / B}$ which can not be obtained within a perturbation analysis. (ii) $\left(v_{s} / v\right) \omega_{B} \ll \omega<\omega_{B}$. Here also only the '-' modes are excited while their nature becomes partially phonon-like as the frequency increases. In this regime, $A_{-} \approx 4 \eta \tilde{B}^{2} \sin ^{2}(\tilde{\omega} / 2)$ and $A_{+}=0$. In particular for $\tilde{\omega}<1$ (i.e., $\omega<v_{s} / L$ ), we get

$$
\operatorname{Re} \alpha(\omega) \approx g \frac{e^{2}}{h}\left(\frac{v_{s}}{v}\right)^{3}\left(\frac{\omega_{B}}{\omega}\right)^{2}
$$

(iii) Finally when $\omega_{B}<\omega$, the ' + ' modes play a significant role and $A_{+}$has singularity near $\omega=\omega_{B}$. Near this singular point, in spite of the phononic nature of the ' + ' modes, significant electric current may flow through its 'diamagnetic' contribution;

$$
\operatorname{Re} \alpha(\omega) \approx g \frac{e^{2}}{h} \frac{|\omega|}{\sqrt{\omega^{2}-\omega_{B}^{2}}}
$$

The singularity of $\alpha(\omega)$ originates from the density of states of the massive boson mode $\omega_{+}(q)$ near $\omega=\omega_{B}$. Similar to Landauer conductance quantization, a quantization rule $\operatorname{Re} \alpha(\omega) \approx g e^{2} / h$ appears in weak coupling regime $\omega_{B} \ll \omega<v / d$.

Important to all the physical consequences discussed in this work is a characteristic frequency $\omega_{B}$ associated with the back-reaction

$$
\omega_{B}=\sqrt{\frac{v_{F} e^{2}}{\rho_{m} \hbar \pi}} B=0.88 \times 10^{-2} B[\mathrm{~T}] \sqrt{\frac{v_{F}[\mathrm{~m} / \mathrm{s}]}{\rho_{m}[\mathrm{~kg} / \mathrm{m}]}} \mathrm{Hz}(19)
$$

where we recovered $\hbar$. As clear in its form, electronelectron interactions do not affect this characteristic frequency. For single-walled carbon nanotubes, the linear mass density is estimated to be $\rho_{m} \sim 4 \times 10^{-13} \mathrm{~kg} / \mathrm{m}$ when its radius is $\sim 4 \mathrm{~nm}$. Using a typical Fermi velocity $v_{F}=10^{5} \mathrm{~m} / \mathrm{s}$, we estimate $\omega_{B} \approx 4.4 B[\mathrm{~T}] \mathrm{MHz}$, which is in the experimentally accessible range.

While we consider electro-mechanical coupling via Lorentz force, capacitive coupling to external gates [2, 13 might be of interest in other contexts. In this case, the coupled charge density and phonon displacement is described by $\sim \int d x u(x) \partial_{x} \theta$. We assumed no external source of mechanical energy dissipation of the wire, which is another subtle issue of electromechanical system [14] and important to clarify the detailed operating conditions of quantum NEMS.

In summary, we outlined a theory for electrons in onedimensional wires in quantum mechanical motion. Quantum mechanical displacement coupled to electric current and the back reaction from electrons give rise to a current-carrying boson mode with an energy gap. The induced electric current by oscillating electric field might reveal the elementary excitations in the system through its frequency dependence. We presented here a nonperturbative regime where the induced electric current has non-trivial frequency dependence.

This work was supported by Korea Reseacrh Foundation (KRF-2003-070-C00020). We thank the partial financial support of BK21 physics research division in Seoul National University where a part of this work was done (K.H.A.) and the Swiss-Korean Outstanding Research Efforts Award Program (H.Y.).

[1] D. Bishop et al., Physics Today 54,No 10, 38 (2001).

[2] P. Kim and C. M. Lieber, Science 286, 2148 (1999).

[3] T. Rueckes et. al., Science 289, 94 (2000).

[4] S. Tomonaga, Prog. Theor. Phys. (Kyoto) 5, 544 (1950).

[5] J.M. Luttinger, J. Math. Phys. N.Y. 4, 1154 (1963).

[6] F. D. M. Haldane, Phys. Rev. Lett. 47, 1840 (1981).

[7] A.N. Cleland and M.L. Roukes, Appl. Phys. Lett. 69, 2653 (1996) ; Sens. Actuators A 72, 256 (1999).

[8] P. Mohanty et. al., Phys. Rev. B 66, 085416 (2002).

[9] H. Onishi et. al.,Nature (London) 395, 780 (1998); A.Y. Yanson et. al., Nature (London) 395, 783 (1998).

[10] See e.g., G. Grüner, Density Waves in Solids, AddisonWesley Publishing Company (1994).

[11] See e.g., G. D. Mahan, Many-Particle Physics, Plenum Press, New York (1990).

[12] C.L. Kane and M.P.A. Fisher, Phys. Rev. Lett. 68, 1220 (1992).

[13] S. Sapmaz et al., Phys. Rev. B 67, 235414 (2003).

[14] K.-H. Ahn and P. Mohanty, Phys. Rev. Lett. 90. 085504 (2003). 